\title{
El momento latinoamericano de Seuil
}

\author{
La colección Cadre vert de Claude Durand y Severo Sarduy \\ (1967-1979)
}

En los años sesenta y setenta, la editorial Seuil publicó algunas de las más destacadas novelas latinoamericanas del período. Paradiso (1966), Cien años de soledad (1967), El mundo alucinante (1965), El obsceno pájaro de la noche (1970), El beso de la mujer araña (1976) son algunos de los títulos que fueron reunidos en el Cadre vert. El "Marco verde", la principal colección de literatura extranjera de la editorial, se distinguía por la sobria portada blanca ceñida por el cintillo verde de sus libros, que retomaba el diseño de Serge Fauchereau de 1958, para la colección de literatura francesa de la casa, Cadre rouge. Vista con la distancia de cincuenta años, la lista compone una impresionante constelación donde cada novela brilla por su propia luz y, por reverberación mutua, ilumina sus vecinas. Su irradiación debe sin duda entenderse como un efecto del llamado boom, si damos al término el sentido restringido de un fenómeno de circulación y difusión de la literatura latinoamericana, con tres polos principales, Buenos Aires, Barcelona y París (Rama 1984: 66; Gras 2000; Steenmejer 2002). Cadre vert fue, con Du monde entier en Gallimard, la principal colección del boom en Francia. La primera dificultad metodológica que plantea su estudio es el hecho de que la colección todavía existe y por lo tanto, y a diferencia de su predecesora La Croix du Sud exclusivamente dedicada a la literatura latinoamericana y concluida a finales de los años sesenta, no se ofrece como un objeto histórico, delimitado por su período de existencia. Los años 1967-1979 se imponen sin embargo como un recorte pertinente ya que corresponden al momento en que la literatura latinoamericana no solo entró en la colección sino que adquirió en ella un protagonismo que no volverá a tener después. Su exploración ocupará las páginas que siguen.

El período circunscrito pone en el centro de atención a dos editores, Claude Durand, quien inició y dirigió la "selección de literatura latinoamericana" de Cadre vert hasta dejar Seuil en 1979 y Severo Sarduy, quien asesoró al primero y luego retomó la responsabilidad del sector hispánico de la casa. La trayectoria del primero nos servirá de hilo conductor para comprender la inserción de la selección latinoamericana en Seuil y en el resto del campo editorial francés y su contexto político e intelectual. En tanto responsable de la selección, Durand debía, por otra parte, hacerse cargo de la negociación y asumir el balance de su

Gersende Camenen, Université Gustave Eiffel

Ә Open Access. (C) 2022 Gersende Camenen, published by De Gruyter. (c) BY-NC-ND This work is licensed under the Creative Commons Attribution-NonCommercial-NoDerivatives 4.0 International License. https://doi.org/10.1515/9783110707557-008 
actividad, de modo que su papel pone de relieve un factor decisivo en la construcción y en la vida de la colección: su naturaleza de empresa comercial, regida por sus leyes propias -la competencia, el cálculo de los riesgos- y las circunstancias que, al igual que el azar o la suerte, incidieron de manera decisiva en la selección de un manuscrito o de un autor y, a la postre, en el perfil de la colección (Spiers 2011: 11; De Diego 2019: 114).

Con Sarduy, esbozaremos la línea editorial de la selección. No sugerimos por ello que la gestión de la colección radicara en una estricta distribución de las tareas - la dirección comercial y administrativa por un lado y la selección de títulos por otro-, al contrario, la colección se presentó como una obra común, y tendremos ocasión de comprobar que los dos hombres compartían un sentido de la estrategia, una manera de ejercer su oficio, es decir un estilo enérgico que influyó en el destino de la colección. Sin embargo, tanto por su origen cubano como por su identificación con la "nueva crítica" y la revista Tel Quel, Sarduy dejó su huella personal sobre la colección. También porque, como miembro de cierta "élite itinerante" del boom, la que Mundo Nuevo congregó alrededor suyo, alimentó su colección con los textos y las ideas que circulaban por las redes transnacionales de la época (Catelli 2010).

\section{Seuil y la literatura latinoamericana}

Nada parecía predisponer a la editorial Seuil a convertirse en el transcurso de los años sesenta en un editor de novelas hispanoamericanas. Fundada en 1930 por un grupo de jóvenes católicos progresistas, la editorial renació en 1945 bajo el impulso de dos hombres, Jean Bardet para la gestión administrativa y Paul Flamand en la dirección editorial. El catolicismo de los orígenes, reforzado por la experiencia de las redes de la Resistencia, seguía siendo, en esos años de posguerra, uno de los pilares de la editorial que publicaba obras de teología y pastoral y se convirtió en el editor de la revista Esprit de Emmanuel Mounier (Serry 2002; 2005). Con el tiempo, el compromiso católico de los comienzos se convirtió en una actitud ética que llevó a Seuil a ser, por sus publicaciones, un actor de la reconstrucción de la "Nueva Alemania", de la edificación de Europa, de la descolonización y, luego, como veremos, del ingreso de América Latina en su órbita.

Desde el principio, Bardet y Flamand ambicionaron convertir Seuil en una grande maison literaria, lo cual implicaba deshacerse de la imagen de una casa confesional y austera esencialmente dedicada a las ciencias humanas. Como lo muestra Hervé Serry, la tarea no fue fácil para los recién llegados al mundo editorial. Desprovistos de una herencia, de las redes sociales necesarias para atraer 
autores como podía hacerlo Julliard, y de una línea vanguardista a lo Minuit, los dos novatos debieron forjarse una identidad inventando sus propios recursos. Supieron desde el principio rodearse de colaboradores de generaciones y sensibilidades muy diversas como el crítico y editor suizo Albert Béguin, precursor de la escuela de Ginebra, el sartreano Jeanson colaborador des Temps Modernes, los católicos Estang y Lesort, el cineasta marxista Chris Marker o François Wahl, editor de Ricoeur, Barthes et Lacan (y compañero sentimental de Severo Sarduy). La diversidad de su comité de lectura era una fuerza para Seuil, ya que relacionaba por capilaridad la joven casa con el medio intelectual y la prensa y otorgaba una gran libertad de acción a sus editores. Esta autonomía, en sintonía con la filosofía personalista de Mounier, aseguraba la convivencia entre los responsables de colecciones (reforzada por su participación en el capital de la empresa a partir de los años sesenta) aunque se hiciera, quizás, en desmedro de una clara línea editorial. Prudencia y adaptación al mercado, autonomía de los editores y necesaria convivencia de las personalidades, son algunos de los sutiles equilibrios de la maison Seuil que, a su manera y en su propio ámbito, intentaron encontrar Durand y Sarduy en la sección hispánica de Cadre vert.

Los fundadores entendieron, por lo demás, que el auge de su editorial dependía de la constitución de un catálogo de literatura. Las oportunidades ofrecidas por el mercado permitieron compensar la ausencia de un catálogo de fondo de la casa: aprovechando el rápido éxito del libro de bolsillo, Seuil se abrió un nicho con la creación de una colección de divulgación dedicada a grandes autores clásicos o contemporáneos. Las pequeñas antologías críticas de "Écrivains de toujours" extendieron la cultura letrada y permitieron, de paso, reclutar autores de Gallimard. Con títulos sobre Sartre, Malraux o Camus, la nueva casa se benefició así, sutilmente, del capital simbólico del editor consagrado. La constitución de un catálogo de literatura extranjera fue otro paso decisivo para el crecimiento y, desde este punto de vista, la presencia de Paul Flamand en Fráncfort en 1949 es una temprana señal del interés de Seuil por desarrollar un sector de traducciones (Serry 2006: 70). La importación de literatura extranjera empezó desde los años cincuenta con las literaturas italiana y alemana. Desde finales de los años cincuenta, la literatura española hizo su aparición en el catálogo, en la colección "Méditerranée”, pensada por su director, el escritor Emmanuel Roblès, pied-noir amigo de Camus y traductor de Lorca, alrededor de temáticas como la "pasión”, la "alegría” y la "tragedia”.

América Latina entra en la editorial en 1958, con un número especial de la revista Esprit que pretendía "iniciar” al lector francés a sus realidades políticas y sociales. Pese a su intención, la introducción del dossier así como gran parte de las contribuciones dibujan equívocamente la imagen exótica de un continente apenas liberado de las garras del imperio español (Tuñón 1958: 307). La 
traducción de literatura latinoamericana empezó unos años más tarde y no dependió, como se verá a continuación, de las mismas redes que la española ni compartió la visión anacrónica del dossier de Esprit. Su aparición en el catálogo correspondió a una nueva época en la historia de la editorial que se convertía para entonces en la segunda "gran casa" del campo editorial y podía diversificar su importación (Simonin 1998: 55). El sector de la literatura extranjera iniciaba una metamorfosis con el desarrollo de las ferias, la presencia de los agentes y aparición en las editoriales de sectores de derechos y adquisición (Sapiro 2019: 81). El ritmo de las traducciones literarias en Seuil se aceleró, pasando de un promedio anual de siete a catorce títulos entre 1960 y mediados de la década siguiente y empezó entonces a dominar el inglés, señal de que la editorial había ganado prestigio y poder de adquisición. La mayoría de los títulos aparecieron en Cadre vert, la colección del Seuil que publicaba a los autores extranjeros contemporáneos. Si bien existía desde 1958, Cadre vert tardó un tiempo en imponerse como una colección, como lo indica, por ejemplo, el que su nombre no figure en los catálogos comerciales destinados a los libreros. Este lento reconocimiento se debe al lugar segundario que ocupaba la literatura en el Seuil de los principios y a la manera de organizar la selección de títulos, confiada a editores especializados en uno o dos ámbitos culturales y que gozaban de cierto margen de acción. Cuando, a mediados de los años sesenta, Durand y Sarduy inician su selección de literatura latinoamericana, la colección estaba instalada en el campo editorial y podía ofrecerle a la vez un estuche y un escaparate.

\section{Durand, el duelista}

La trayectoria de Claude Durand (1938-2015) se inscribe en una tradición republicana e ilustra, por otra parte, el ascenso de la figura de editor en el campo intelectual francés. Su llegada al mundo hispanoamericano a mediados de los años sesenta fue, como veremos, fruto de circunstancias a la vez personales y profesionales. De origen modesto, Durand se formó como maestro en una escuela normal. La literatura y la política fueron las dos pasiones que el joven institutor cultivó conjuntamente, subraya su biógrafo François Chaubet. Se inició en la segunda en los años en que Pierre Mendès France entusiasmaba a los sectores progresistas con su alternativa socialista al entonces imperante partido comunista. De su juventud meritocrática, el maestro mendesista heredó la ambición de elevar las conciencias por el acceso a la cultura y, con el tiempo, extendió su civismo republicano a la preocupación por el injusto reparto mundial de los frutos del progreso técnico, encontrando su formulación en el "tercermundismo" acuñado en 
1952 por el demógrafo Alfred Sauvy y que empezaba a circular en los medios progresistas.

En 1957, Durand conoció a Jean Cayrol, poeta, novelista, editor que "gozaba entonces del reconocimiento de los distintos actores del campo intelectual, desde los existencialistas, los personalistas cristianos y hasta la vanguardia del Nouveau Roman” (Basuyaux 2004). Cayrol publicó el primer texto literario de Durand en Ecrire (1956-1966), su revista-colección dedicada a la publicación de jóvenes escritores como Philippe Sollers, Denis Roche o Kateb Yacine y apoyó luego la publicación en Seuil de sus siguientes novelas, alabando su estilo "un poco barroco” y soñador. Entre 1960 y 1965 colaboraron en la realización de cortometrajes y de un largometraje, Le coup de grâce (Premio Jean Vigo) y publicaron un ensayo-manifiesto sobre el cine, Le droit de regard, en el que exponían un proyecto estético con ambición ontológica, vanguardista en su formulación -aunque lo fuera menos en su realización- ya que consistía en "desfamiliarizar” el mundo restituyéndole su profundidad original y su radical novedad. De sus años de formación con Cayrol, Durand conservó una facultad de asombro ante lo real, la apreciación del poder de la imaginación, en otras palabras, lecciones literarias y vitales que en cierta forma lo predisponían al descubrimiento de la literatura latinoamericana. A su lado, aprendió también los entresijos del oficio de editor (la lectura rigurosa de los manuscritos, la oportunidad dada a los nuevos talentos, la capacidad de aceptar rápidamente un texto que le gustaba) y afinó el olfato que le permitiría hacerse con Cien años de soledad y ganar su legitimidad profesional.

1965 fue sin duda un año decisivo para Durand y su encuentro con el mundo hispanoamericano. Entró en la plantilla de Seuil para secundar a Monique Nathan, especialista reconocida de literatura anglo-sajona pero a raíz de una reorganización interna, fue nombrado responsable de la literatura hispanófona en Cadre vert, hasta entonces representada por autores españoles (Luis Goytisolo, Camilo José Cela, Luis Martín-Santos). El mismo año se casó con Carmen Perea Jiménez (1937-2016), nacida en La Habana en una familia acomodada que se había exilado a España en la época de Fulgencio Batista y a quien Durand había conocido en el rodaje de Le coup de grâce. Con Carmen, el editor se hizo traductor, de Cien años de soledad y luego de Fuera de juego. Son escasos los datos que sobre su colaboración tenemos; Carmen "se lanzaba" en la traducción que luego "retomaba" Claude, resume escuetamente Alain Beuve-Mery en el obituario de la cubana. Es de pensar que la presencia de Carmen, "apreciada por los autores" de su marido fue decisiva en su trayectoria editorial y su papel en la difusión de la literatura latinoamericana -fue traductora de Isabel Allende- merecería probablemente una investigación aparte.

Entre 1965 y 1979, fecha en que abandona Seuil por Grasset, Durand operó la selección de las obras latinoamericanas de Cadre vert, así designadas en la 
contratapa de los libros y que a continuación reproducimos. No cabe duda de que la relación profesional que Durand entabló con Carmen Balcells haya desempeñado un papel decisivo en la constitución del catálogo. En una entrevista publicada en 1999, Claude Durand recordaba que antes de ser la "agente poderosa que llegó a ser [. . .], [Balcells] quería su opinión sobre todo” y leía con avidez los largos informes de lectura que el joven editor le mandaba. De modo que, cuando llegó el manuscrito de Cien años de soledad a las editoriales francesas, mientras que en Gallimard, Roger Caillois, el director de La Croix du Sud respetó "los usos de la casa según los cuales se tardaba seis meses en contestar"; Durand lo compró inmediatamente "por 5000 francos” (Lindon 1999: 7). La anécdota ilustra un cambio de época en las maneras de practicar el métier de editor. Con la llegada de los agentes, se había convertido en un combate de esgrima del cual, por definición, no se salía siempre victorioso. En 1974, Carmen Balcells comunicó a Durand que El otoño del patriarca no saldría en el 27 de la rue Jacob. En una nota interna, Durand expuso a Paul Flamand el conflicto latente que los oponía a la agente barcelonesa: desde que "había elegido" a Seuil para Cien años de soledad, la mamá grande exigía de la editorial "la representación exclusiva para la lengua española” (Chaubet 2018: 90). En la misma nota, midiendo la fuerza de su contrincante, Durand cuestionaba la supervivencia de una sección hispánica que no se alimentaría de la cantera Balcells:

\footnotetext{
¿Podremos mantener un sector hispanoamericano después de una ruptura total con el agente que representa a la casi totalidad de los escritores importantes de esta literatura? [. . .] la respuesta es negativa. Ya hemos reunido a todos los autores que no dependen de C. Balcells: Sábato, Puig, Lezama Lima, Arenas y al propio Severo. Podemos contentarnos con esto, pero no es "hacer vivir" un sector y en este caso, Severo y yo preferiríamos abandonar el juego y entregar este sector, reducido a las proporciones del sector griego, a un responsable del ámbito extranjero (Chaubet 2018: 90).
}

La preocupación de Durand se hizo realidad y no solo El otoño, y García Márquez, "se fueron" a Grasset sino que la selección de literatura latinoamericana del Cadre vert no incluyó a nuevos autores después de 1974 y hasta el final del período aquí estudiado. Si bien otros factores, estéticos e intelectuales, pesaron en las decisiones de Durand y Sarduy, es evidente que las reglas del juego editorial, y concretamente el poder de la agencia Balcells trazaron, no sin cierta aspereza, los límites de su perímetro y de su catálogo. Durand, amaestrado en el arte de bregar por los intereses de su casa, lo entendió perfectamente, y es de suponer que la frustración ocasionada por l' "Affaire Gabo" contribuyó a orientarlo hacia otros proyectos (Díaz Martínez 2021).

En 1968, había creado "Combats", una colección de ensayos y libros de actualidad que, con más de cien títulos publicados en diez años de existencia, 
“fue la diagonal de las contestaciones que agitaron el mundo entre 1965 y 1975: las luchas armadas sudamericanas y palestinas, la revolución cultural china, las esperanzas de un comunismo democrático en el sur de Europa, la disidencia en la URSS” (Chaubet 2018: 109). Combats nació de un interés personal de Durand por Latinoamérica pero se inscribía por supuesto en una coyuntura política que lo superaba, la del 68, en la cual el continente latinoamericano se convirtió, por unos años, en un foco de observación y, para los sectores intelectuales progresistas, en una fuente de inspiración y esperanza. En su crónica de los años sesenta, el historiador Michel Winock apunta:

Cuando en enero de 1968, Claude Durand lanzó su colección “Combats”, sus primeros títulos venían del continente elegido por la Revolución: le Journal d’un guérillero, luego Ecrits et Paroles de Camilo Torres y, en mayo de 1968, la biografía de Che Guevara por Ricardo Rojo. Los braseros de América latina no se podían importar al Viejo Mundo pero avivarían ahí el espíritu de rebeldía; las barricadas de mayo no tardarían en manifestarlo (Winock 1987: 224).

Con Combats, Durand se aventuraba en los terrenos arriesgados de la edición política, pero lo hacía desde una posición política que lo distinguía de los editores militantes que tradicionalmente la frecuentaban, como un François Maspero que reunía en su librería La joie de vivre toda la juventud y la intelligentsia libertaria y publicó en su revista Partisans al Che, Fidel Castro y Régis Debray entre otros autores de la izquierda radical y revolucionaria mundial (Hage 2011). La línea política de Combats era "comprometida sin ser sectaria, filocomunista (uno de sus autores principales era Régis Debray) sin ser indiferente a la crítica del comunismo (la de los disidentes soviéticos)" (Chaubet 2018: 110). Y reflejaba sin duda la inclinación del propio Durand. Ante el comunismo, es decir la cuestión que se había impuesto a su "generación" y obligaba a sus miembros a definirse en función de ella (Winock 2011: 7), Durand había elegido una izquierda no radical, la de su juventud mendesista, que siguió buscando su camino en los años setenta y desde la cual emergió una crítica a los comunismos instituidos. Durand, por lo demás, entró en Seuil en el "momento de gracia” de la edición: con el desarrollo de las colecciones de ciencias humanas, la edición empezaba a ejercer un verdadero magisterio intelectual sobre la sociedad, sustituyendo a una universidad que aparecía cada vez más anquilosada, como lo demostró la sonada polémica Barthes-Picard en 1965. Apoyadas, por lo demás, en el creciente poder de los medios de comunicación masiva, las editoriales iban creándose nuevos públicos a quienes proponían instrumentos intelectuales para comprender un mundo en plena efervescencia (Dosse 2014: 6). Durand edificó sus colecciones en este ecosistema, en el cual se movía a sus anchas: los libros de Combats se dirigían a un público más amplio que el de los círculos politizados, el público de una inci- 
piente "cultura de la actualidad" que otras editoriales también intentaban captar con la creación de colecciones similares (Chaubet 2018: 116) ${ }^{1}$.

Como puede apreciarse por la lista de sus publicaciones, Combats no alejó a Durand de América latina ni lo apartó de sus preocupaciones literarias, al contrario, agudizó su mirada sobre el continente y su comprensión de los públicos lectores. La gestión paralela de las dos colecciones ilustra por lo demás un rasgo de los años sesenta y setenta: la política y la literatura se compenetraban en el campo intelectual y editorial de finales de los años sesenta, tanto en Francia como en América latina, donde dicha politización fue, según Claudia Gilman, el rasgo definitorio de la época. Le Cadre vert no quedó fuera de esta politización como lo indica la emblemática publicación de Hors Jeu de Heberto Padilla en 1969. Nótese que, para la fecha, los disidentes soviéticos empezaban a imponerse en el catálogo de Combats ${ }^{2}$ y que desde 1970 Durand supervisaba la edición de los textos Soljenitsyne para, cuatro años después, dedicarse a la gestión exclusiva de los derechos internacionales de sus obras (Durand 2011: 15-17). No es exagerado pensar que el "agente" del mayor disidente soviético sintiera una especial empatía por los escritores cubanos que integraban su catálogo. En este caso, como en otros, tanto el complejo perfil de Durand como las circunstancias profesionales y políticas en que ejerció su oficio moldearon en gran parte el catálogo latinoamericano de Cadre vert. Sin embargo Durand, aunque solitario, no trabajaba solo, aun no era "el emperador Claude" empoderado por sus éxitos y temidos por sus colaboradores y rivales (Dosse 2014: 73). Se había rodeado de un equipo de lectores en el cual destacaban el escritor español Xavier Domingo, el universitario Alain Rouquié, el traductor Didier Coste y por supuesto Severo Sarduy, cuya mirada crítica delineó los contornos de la selección.

1 Dichas colecciones eran "Contestation” en Robert Laffont, dirigida por Max Gallo, "Lutter" en Stock, "La France Sauvage” en Gallimard.

2 Entre 1968 y 1976, se publican 14 libros dedicados a América latina, 15 sobre la disidencia en la Unión soviética, 21 sobre temas de actualidad francesa, 13 de teoría y reflexión crítica (entre los cuales figuran los de Régis Debray, en parte dedicados a América latina) y 25 sobre otras regiones del mundo. Los títulos latinoamericanos se concentran en los primeros años mientras que los de la disidencia soviética siguen una curva ascendente a lo largo de los años de existencia de la colección. 


\section{7-1979, el momento latinoamericano de Seuil}

La lista que proponemos a continuación se compone de los títulos de literatura hispanoamericana publicados durante los años en que Claude Durand dirigió la selección de literatura latinoamericana de Cadre vert y que constituyen en cierto modo una edad de oro para la literatura latinoamericana en Seuil, durante el cual se la consideró y presentó al lector como un conjunto autónomo. La llegada de Durand en la sección hispánica de la colección de literatura extranjera abre una etapa nueva y hasta cierto punto fundacional, con la publicación en 1968, de Cent ans de solitude, el "batacazo" editorial de Durand y como lo señala el hecho de que, a partir de 1967, aparezca en la contratapa de cada libro la lista de los otros títulos publicados en la colección, encabezada por la mención "Euvres de la littérature latino-américaine choisies par Claude Durand et Severo Sarduy". Esta presentación paratextual producía el doble efecto de dar al conjunto de libros reunidos la cohesión de una suerte de pequeña colección dentro de la colección de literatura extranjera y de asentar la autoría editorial de los seleccionadores ${ }^{3}$. A partir de 1979, cuando Durand deja Seuil y hasta 1990, Sarduy dirige la sección hispánica de Cadre vert; publica títulos de los autores de la selección hecha con Durand pero no los conserva a todos ni extiende de manera decisiva el catálogo latinoamericano. A partir de los años ochenta, Cadre vert publica más títulos españoles que latinoamericanos, con las novelas de Juan Goytisolo y luego Eduardo Mendoza y Manuel Vázquez Montalbán sobre todo ${ }^{4}$. El cambio se vuelve explícito, ya que a partir de 1979, la lista pasa a titularse "Éuvres traduites de l'espagnol aux éditions du Seuil choisies par Severo Sarduy” e incluye no solo a los autores españoles publicados a partir de la fecha sino también títulos anteriores incluso al año 1965. Con esta inclusión retrospectiva y la nueva orientación editorial, la literatura latinoame-

3 La lista incluye también libros editados antes de 1965 y que por lo tanto no pudieron ser elegidos por Durand y Sarduy: Buriti (1961) y Les nuits du Sertão (1962) de João Guimarães Rosa. Se incluye también Gestos (1963), la primera novela de Sarduy. Esta inclusión refuerza la imagen de 'catálogo' de las obras seleccionadas en detrimento de cierto rigor ya que la autoría de Durand y Sarduy aparece como retroactiva.

4 Bajo la gestión de Sarduy, se publican Le jeu des décapitations (1984) y Oppiano Licario (1991) de José Lezama Lima, La plantation (1983), Arturo l'étoile la plus brillante (1985) Encore une fois la mer (1987) de Reinaldo Arenas. Y entran en el catálogo La Havane pour un Infante défunt (1985) de Guillermo Cabrera Infante, Nouveaux contes froids (1988) de Virgilio Piñera, Parade d'amour (1989) y Les apparitions intermittentes d'une fausse tortue (1990) de Sergio Pitol. Tras un interludio con Seuil, las novelas de Manuel Puig vuelven a Gallimard y las siguientes de Donoso se publican en Calmann-Lévy. 
ricana se diluye en un conjunto hispánico y subraya, retrospectivamente, el carácter excepcional del período 1967-1979.

- Arenas, Reinaldo, Le monde hallucinant, traduit de l'espagnol par Didier Coste, 1969

- Le puits, traduction de l'espagnol Didier Coste, 1973

- Le palais des très blanches mouffettes, traduit de l'espagnol par Didier Coste, 1975

- Borges, Jorge Luis, Evaristo Carriego, traduit de l'espagnol par Françoise Rosset, préface d'Emir Rodríguez Monegal, 1969

- Donoso, José, L'obscène oiseau de la nuit, traduit de l'espagnol par Didier Coste, 1972

- García Márquez, Gabriel, Cent ans de solitude, traduit de l'espagnol par Claude et Carmen Durand, 1968, prix du meilleur livre étranger

- Guimarães Rosa, João, Hautes plaines, traduit du brésilien par Jean-Jacques Villard, 1969

- Lezama Lima, José, Paradiso, 1971, traduction de Didier Coste

- Padilla, Heberto, Hors-jeu, 1969, traduction de Claude et Carmen Durand

- Puig, Manuel, Les mystères de Buenos Aires, 1975, traduction de Didier Coste

- Le baiser de la femme-araignée, 1979, traduction d'Albert Bensoussan

- Sábato, Ernesto, Alejandra, traduit de l'espagnol par Jean-Jacques Villard, préface de W. Gombrowicz, 1967

- L'ange des ténèbres, 1976, traduit de l'espagnol par Maurice Manly, prix du meilleur livre étranger

- Le tunnel, 1978, traduit de l'espagnol par Michel Bibard5.

- Sarduy, Severo, Ecrit en dansant, 1967, traduit du cubain par Etienne Cabillon, Claude Esteban et l'auteur

- Cobra, 1972, traduit de l'espagnol par Philippe Sollers et l'auteur, prix Médicis du roman étranger.

La lista invita a formular una serie de observaciones y las primeras son de orden cuantitativo. El número de títulos publicados, dieciséis libros en catorce años, es relativamente limitado, lo cual da a la selección unas proporciones modestas. Sin embargo, si solo se consideran los primeros años, el catálogo no es tan exiguo: diez libros entre 1967 y 1972, e incluso siete libros entre 1967 y 1969, siendo el último un año pico con cuatro publicaciones. El ritmo disminuyó de manera muy sensible después de 1972, con solo seis libros publicados entre

5 Una primera traducción de la novela de Ernesto Sábato, a cargo de Carmen Sangrado, fue publicada en La Croix du Sud en 1956. 
1973 y 1979, lo cual parece confirmar que el pulso perdido con Balcells incidió en la posibilidad de contratar a nuevos autores. Sin contar que los títulos publicados después fueron de autores de la casa (Arenas y Sábato) y de un autor (Puig) que no había confiado su representación a la agencia Balcells. Atribuir la responsabilidad del destino de la colección, de su relativo declive, al juego de esgrima con Balcells sería sin duda exagerado -también llegaba la crisis del petróleo y una baja general de la edición francesa- pero es cierto que este primer examen dibuja la imagen de una colección truncada, de un impulso editorial trabado por las circunstancias.

\section{Lo "actual": una estrategia, una línea, un estilo}

Si bien la colección estaba constituida casi exclusivamente de novelas contemporáneas ${ }^{6}$, difícilmente podía constituir una línea editorial ya que publicar novelas no representaba ninguna originalidad para una casa generalista como Seuil. Y, precisamente, la necesidad de diferenciar la colección en el campo editorial fue lo que modeló la selección de Durand y Sarduy. La suya fue una apuesta por "lo actual", calificativo que vuelve una y otra vez en el discurso editorial y que, pese a sus contornos algo borrosos, nos parece resumir con acierto la selección de Durand y Sarduy, su proyecto y su manera de editar, en otras palabras, el espíritu que la animaba.

6 Desde este punto de vista, Evaristo Carriego y Hors Jeu aparecen como las excepciones que confirman la regla y, de hecho, la publicación de cada uno sigue su propia lógica editorial. Con la traducción de Evaristo Carriego se capta a un autor clásico destinado a consolidar un catálogo en busca de su legitimidad. El hábil prefacio de Monegal confirma la estrategia al hacer de Evaristo Carriego una obra clave de Borges. Desde su primera frase, "todo lo que Borges toca se convierte en ficción”, el crítico uruguayo no solo establece una continuidad genérica entre la biografía del poeta porteño y el libro emblemático de la poética narrativa de Borges sino que traza una línea simbólica entre la selección de Cadre vert y la colección de La Croix du Sud que, como se sabe, se iniciaba en 1951 con la publicación de Fictions. No solo eso, sino que Monegal sugiere leer Evaristo Carriego como una suerte de autofiguración del propio Borges - "todo lo que toca Borges se convierte en Borges” escribe en conclusión-. Su interpretación, sin duda válida, convierte un libro menor y a priori poco atractivo para el lector francés de a pie en una perla de imprescindible lectura, a la vez cifra y figura de su autor (Rodríguez Monegal 1969: 5-8). La operación de prestigio se completa, con la publicación en 1970 de Borgès par lui-même, a cargo del mismo Monegal, y que es, por lo demás, el primer número de la colección "Écrivains de toujours” dedicado a un escritor de lengua española. 
Una descripción de este proyecto y de su estilo se encuentra en una encuesta sobre la edición de literatura latinoamericana en Francia publicada en Mundo Nuevo en 1968:

[Seuil] opera según un criterio definido: selección de los autores en función de un criterio que podríamos decir de orientación estética; en consecuencia, -menos escritores que Gallimard por ejemplo-, pero tratando de sacar mejor partido de la tendencia estética de los mismos -más al gusto "actual" del público francés-, y contando mucho con la promoción, ocupándose de cerca de sus autores (Bareiro Saguier 1968: 65).

Se percibe el entusiasmo y la determinación de Durand y Sarduy presentados incluso, quizás un poco pomposamente por Bareiro Saguier, como dueños de "toda una política de publicación de los últimos autores latinoamericanos” (1968: 57). Este tono algo petulante así como las consignas de la "política" enunciada, adecuarse "al gusto actual” y "operar según un criterio estético", revelan un deseo estratégico de diferenciar la selección de Cadre vert de dos otros actores del campo, un predecesor y un competidor directo, ambos de Gallimard y designados sin muchos rodeos en la encuesta. Afirmar que se seguía un "criterio estético" era en efecto una manera de distanciarse de la línea documentalista y etnográfica de La Croix du Sud. Desde mediados de los años sesenta, la colección de Caillois, edificada sobre la idea de una representatividad cultural de las obras literarias, aparecía en efecto como una "empresa anacrónica" que había perdido su poder consagratorio de la literatura latinoamericana en el campo literario francés donde iban emergiendo voces, como la de Maurice Nadeau, para reclamar un necesario aggiornamento (Guerrero 2018: 206).

La razón del declive de la colección de Caillois era también comercial, "las ventas no funcionan", reconocían los responsables en la misma encuesta (Bareiro Saguier 1968: 65). Una colección especializada ya no tenía razón de ser en un mercado diversificado en el que el lector "apabullado por los centenares de otras novelas que aparecen no demuestra un interés especial por la literatura latinoamericana" (Bareiro Saguier 1968: 66). Esta nueva realidad económica fue otra circunstancia que, como la batalla perdida contra Balcells, influyó decisivamente en las proporciones modestas de la selección latinoamericana de Cadre vert. Lo que Durand y Sarduy presentaban en la encuesta como una política de autores, selecta y cuidadosa, era (también) la aplicación de un modelo económico que buscaba "sacar el mejor partido de la tendencia estética de los mismos" y crearse un nicho en un mercado restringido en el cual el verdadero competidor, del que los editores querían distinguirse en la encuesta, era Du monde entier, la colección de literatura extranjera de Gallimard que empezaba a publicar a escritores "jóvenes" (Bareiro Saguier 1968: 65) y otros que, como Carlos Fuentes, huían del "gueto" de La Croix du Sud (Monegal 1966: 21; Louis 2014: 80). 
Sin embargo, aunque estratégica, la apuesta de la selección de Cadre vert por una literatura "actual" y por un número restringido de autores fue el fruto de una lectura interpretativa de las novelas y reducirla a una mera operación comercial sería un error. Durand y Sarduy elaboraron un discurso crítico que impuso un perfil al conjunto de los libros que seleccionaron, un discurso que, si bien no ignoraba la singular estética de cada novela o escritor, tendía a crear su propia colección como un objeto autónomo. Este discurso no se encuentra formulado programáticamente en una "declaración de intenciones" de la colección sino que emerge del amplio y disperso corpus paratextual constituido por las contratapas, los folletos publicitarios, las presentaciones de los libros destinadas a los profesionales de la edición ${ }^{7}$, reflejos más o menos filtrados de los documentos internos de la editorial como son los informes de lectura y la correspondencia profesional, consultables en los archivos de Seuil que se conservan en el IMEC. La multiplicidad de estos materiales, su heterogeneidad discursiva y, por otra parte, la evidente singularidad estética de cada autor de la selección, vuelven difícil la tarea de definir una lectura editorial general. Quizás porque, lo que se enunciaba, dispersa pero repetidamente, no era un programa. Considérese lo que sigue como un intento de delinear los borrosos contornos de "lo actual".

La novela "actual”, la novela de la selección de Cadre vert, se perfilaba como un apogeo, es decir como, a la vez una suma de la historia y la cultura occidentales, y no solo latinoamericana, y una culminación del género novelesco. Lo actual no era lo simplemente nuevo, la última tendencia, sino un punto de llegada. Cien años de soledad es, según se lee en su contratapa, “el teatro gigante donde los mitos engendran a los hombres que a su vez engendran los mitos, como en Homero, Cervantes o Rabelais"; Paradiso "es tanto el Conocimiento como lo que fue conocido [y se encuentra] en la encrucijada de todos los mundos, las creencias y las civilizaciones”; El ángel de las tinieblas es "tanto "la novela total" de una crisis espiritual y social como la exploración y la culminación de una “crisis de la novela” que la refleja” (27 rue Jacob, n¹97, marzo de 1976). Se alegará que dichas novelas son en sí sumas, y que el argumento editorial era, por lo tanto, mera descripción del proyecto poético del escritor. Pero el calificativo de "novela total" se aplica a otras novelas que no exhiben esta ambición. Es el caso de El mundo alucinante, descrita en su contra-

\footnotetext{
7 Seuil publicó, casi desde su fundación, un "boletín mensual de informaciones" que llevaba por título 27 rue Jacob, referencia a la famosa dirección de la sede parisina de la editorial. En sus páginas, se encuentran artículos de presentación de los libros publicados, resúmenes, recortes de prensa, fotos de las tapas de los libros y de los escritores de la casa pero también datos sobre las ventas de los derechos de los libros de Seuil, sus ventas en el extranjero, algunos artículos de fondo sobre las "actualidades" de la literatura y de las ciencias humanas.
} 
tapa como "biografía imaginaria, cuento picaresco, fábula filosófica, fresco histórico, a la vez grave y burlesco, alucinante de verdad e invención, fantásticamente realista, realmente fantástica”. El mundo alucinante se presentaba al lector como una novela polifacética y versátil y ofrecida a lecturas múltiples que no agotaban las posibilidades del modelo genérico de la novela. Este ejemplo muestra, como los precedentes, que una característica del discurso editorial de la colección era su notable ambición crítica.

Dicha ambición lo llevaba incluso a dibujar un horizonte utópico, un más allá del género novelesco y de la literatura, en general y no solo de la latinoamericana, hacia el cual los libros de Cadre vert apuntaban. Semejante horizonte aparecía en la crítica latinoamericana del boom pero en el caso de Seuil, este discurso llevaba la marca de la "nouvelle critique”, de un Sarduy inmerso en Tel Quel y auditor asiduo de los seminarios de Barthes en la École des Hautes Études en Sciences Pratiques, como lo ilustra el artículo que dedicó a Cien años de soledad, titulado "La escritura autónoma” y publicado en la Quinzaine littéraire en 1968. Desde un andamiaje autoreflexivo y autónomo, Sarduy veía en Cien años de soledad una ruptura "con la una tradición realista" latinoamericana que entendía la literatura como un modo de "expresión” de contenidos exteriores. Con la novela de García Márquez, afirmaba, la literatura, liberada de su función representativa, se vuelve "escritura”, y "la literatura”, que es "sucesión de variantes y diseminación”, "se practica aquí como intertextualidad”, el texto se descifra, propone Sarduy siguiendo la vía abierta por Barthes en su seminario sobre Sarrasine, como un "código de papel” (Sarduy 1968: 3-4). Como puede apreciarse en este texto, Sarduy ofrecía a las novelas de la colección un marco interpretativo muy actualizado que propulsaba la literatura latinoamericana seleccionada por Cadre vert en la punta más avanzada del campo editorial francés.

Se puede argüir que el artículo de La Quinzaine comparte con su objeto las cualidades que le atribuye, y por lo tanto constituye un manifiesto crítico relativamente aislado en el discurso editorial. Al reflejar la estética personal de Sarduy, entonces en plena formulación, proponía la versión más radical del "criterio estético” que presidía la selección de los títulos en Cadre vert. Y, por esta razón, estaba en cierto punto en desfase con su objeto, una novela que, al igual que las mayorías de las novelas del boom, no rompía del todo el marco realista. Pero como caso-límite, y precisamente por este desfase, el artículo de La Quinzaine devela la intención del discurso editorial, la cual se entiende a la luz de los movimientos internos de Seuil que, desde los años sesenta, iba multiplicando las creaciones de revistas, Tel Quel o Change, y colecciones como "Fiction \& Cie" para abrirse paso por el codiciado terreno de la literatura de vanguardia (Forest 1995: 55-70; Nadeau 1970: 164-173; Parinet 2004: 403; Simonin 1998: 66-69). 
La novedad formal no era sin embargo el único criterio de selección en Cadre vert. El examen de los paratextos muestra que no se desatendían los temas abordados en las novelas ni su inscripción en una tradición o un contexto nacionales. Así, por ejemplo, la faja que ceñía Le puits (traducción de Celestino antes del alba) incitaba a leer el libro de "una infancia cubana” y en la contratapa el énfasis se ponía en la nacionalidad y el proyecto arqueológico del escritor de "elaborar una Historia desde los tiempos inmemoriales" de la isla. La invitación a descubrir una época, una cultura, un país seguía siendo un criterio de selección porque era -y sigue siendo- un argumento para atraer al lector hacia una colección de literatura extranjera. La presentación paratextual de Alejandra, al subrayar la novedad de las técnicas narrativas de Sábato e invitar al lector a conocer los "secretos de la sensibilidad sudamericana", es un buen ejemplo del equilibrio que el discurso editorial buscaba. Por mucho que quisiera aplicar a las novelas un criterio de literariedad "pura" o de ficcionalización radical, se imponía la necesidad de satisfacer la curiosidad del lector por otras culturas, un deseo que hace de la literatura traducida una empresa de funambulista y que se fustiga a menudo injustamente como mero exotismo.

Línea de lectura, lo “actual” debe entenderse también (o, tal vez, sobre todo) como una manera de encarnar la figura de editor. De presencia discreta trabajando en la sombra, el editor pasaba a ser un actor de primer plano. Los paratextos son el lugar ideal para comprobar este cambio y las contratapas de la colección ofrecen de hecho un verdadero escenario al editor. Es el caso de El mundo alucinante. La apreciación de la novela de Arenas como obra proteiforme y suma de géneros narrativos se presenta explícitamente como una lectura del editor que precisa al lector que "esta obra en la que el propio autor no ve nada más que un relato de aventuras es sin embargo un poco más que una novela”. Al dejar las huellas de su enunciación en la contratapa, el editor sale aquí de las bambalinas y rompe la ilusión de un encuentro a solas entre el lector y su libro. Se figura asumiendo los diferentes papeles que le corresponden a lo largo del trayecto que conduce del texto al libro, el del mentor que revela al escritor el sentido y el valor de su propio trabajo y el de crítico que enseña al lector a interpretar el texto. De este modo, al sacar a la luz el trabajo de trastienda -la selección de los manuscritos, su tasación, la elaboración de un discurso crítico que modela el texto y ofrece incluso las claves hermenéuticas de su lectura- los autores de la contraportada hacían emerger el mundo humano y profesional que rodea los libros.

Durand y Sarduy asumieron otra función de este mundo, la de agentes de promoción de sus libros, en un mercado diversificado en el que acompañar al libro iba siendo una tarea indispensable. No escatimaron esfuerzos en este ámbito y no extraña por lo tanto que la encuesta publicada en Mundo Nuevo haga hincapié en este aspecto. En efecto, el artículo de Sarduy sobre Cien años de 
soledad no fue un caso aislado sino un ejemplo de una práctica que se había vuelto frecuente para las novelas de Cadre vert: el editor (o los editores como en el caso de Paradiso) el traductor o un lector de la editorial se hacían reseñistas de sus propios libros (Guerrero 2021). La práctica no era nueva, pero la reforzaba la compenetración de la prensa y del mundo editorial, acentuada con la aparición de revistas nuevas especializadas (La Quinzaine littéraire y Le magazine littéraire, ambos fundados en 1966) o de los semanarios de nuevo corte dirigidos a las clases medias profesionales, como L'Express creado en 1953 y sobre todo Le Nouvel observateur en 1964. Seuil por lo demás iba desarrollando una política comercial que adaptaba el libro a los modos de consumo cultural de estas mismas clases; es así como Paradiso, "una novela fabulosa" y Le puits, "una infancia cubana", se recomendaban en el boletín de la editorial, 27 rue de $J a c o b$, y en el folleto comercial de "Lecturas de verano" ilustrado con la fotografía de una playa y distribuido a los libreros y profesionales del sector. De esta forma, adecuando su discurso a los formatos y géneros de diferentes publicaciones y soportes, los editores $-\mathrm{y}$ los otros vectores del discurso editorial ${ }^{8}$ - no solo respondían al "gusto actual", como lo formulaba la encuesta, sino que lo iban creando a través de una red de revistas, periódicos y de una serie de figuras transmisoras de su discurso.

"Lo actual" de Cadre vert era un estilo editorial brillante y enérgico, a la vez pragmático y ambicioso que en parte se explicaba por el recorrido y la personalidad tanto de Durand como de Sarduy. Este estilo correspondía, por otra parte, a una coyuntura histórica favorable, un "momento de gracia" de la edición que ya evocamos y que propició el ascenso de una figura omnipresente. El editor "actual” era a la vez un mentor, un hermeneuta, un crítico, un publicista y un prescriptor de gusto.

\section{Afinidades electivas y selectivas: Cadre vert y Mundo Nuevo}

No hace falta haber transitado mucho por la crítica latinoamericana de los años sesenta y setenta para detectar en la lista de autores de Cadre vert y en la línea editorial que intentamos esbozar las huellas de Emir Rodríguez Monegal. Dicha línea y el espíritu que la animaba deben mucho a las vínculos personales y pro-

8 Haría falta detenerse en el papel de los traductores, periodistas, lectores editoriales en la promoción y modulación del discurso editorial. En el caso que nos interesa, el periodista JeanMichel Fossey, el escritor y traductor Didier Coste, por ejemplo, por su intervención en la prensa para el primero o su lectura interna para el segundo, invitan a redefinir los contornos de la función editorial. 
fesionales que unieron a Severo Sarduy y al crítico uruguayo. No es ningún secreto que Sarduy fuera un colaborador regular de la revista fundada en julio de 1966 en París ni que Sarduy, y su obra, encarnaran el ideal de la novela del lenguaje que Monegal desarrolló en la última parte de su ensayo El boom de la novela hispanoamericana de 1972. Es incluso probable que el cubano haya sido el autor con más publicaciones en Mundo Nuevo ${ }^{9}$. El propio Sarduy resumía, en una carta dirigida a Keith McDuffie en 1983, la manera en que Monegal amplió su conocimiento de la literatura latinoamericana y modernizó su acercamiento a ella. Con su habitual encanto, Sarduy se describía como una "emanación”, “una creación literaria” del crítico uruguayo (Gras 2020: 20). Es tentador, por lo tanto, imaginar a Monegal como un editor secreto de la colección, un director que entre las bambalinas movía los hilos, y prestarle, una vez más, un poder oculto (Gilman 2003; McQuade 1992; Morejón 2017; Mudrovic 1997). Pero al hacerlo, no solo caeríamos en una comodidad intelectual engañosa que busca una única causa a un fenómeno complejo, sino que restaríamos importancia al papel que, como vimos, desempeñó Durand en la configuración de la selección. En las apretadas líneas que siguen, nos limitaremos a señalar los puntos de conexión entre la revista y Cadre vert y a someter al examen de algunos textos la hipótesis de un Monegal mentor de la colección.

La manifestación más visible de los vínculos que unían Cadre vert con Mundo Nuevo es la aparición en la revista de todos los autores seleccionados en Seuil (con excepción de Padilla), trátese de la publicación de un adelanto de una novela por salir en la editorial o de una reseña, una entrevista (con Ernesto Sábato, hecha al alimón por Sarduy y Monegal) o incluso, como en el caso de Lezama, de un número homenaje, dirigido por Sarduy. Mundo Nuevo publicó extractos de Cien años

9 Se encuentran nada menos que siete artículos de su mano -tres sobre arte y cuatro de crítica literaria- en los que se va gestando su teoría neo-barroca, una estética que considera la escritura -literatura y artes plásticas- como una continua superficie espejeante de signos que, al desplegarse, teatraliza el artificio del mundo. Los artículos abren los escenarios donde se da este teatro del artificio (el erotismo, la pintura abstracta), indagan sus orígenes hispánicos (la tradición barroca) y enuncian los postulados teóricos que lo articulan a la modernidad (el método estructuralista engrosado por el psicoanálisis), es decir que exponen las coordenadas de un sistema muy sofisticado que ya aparecía en ciernes en la entrevista que le dedicó Monegal (Monegal 1966b). Si a estos textos se añaden la traducción del famoso artículo de Roland Barthes sobre la "faz barroca de Sarduy", la reseña de Donde son los cantantes de Claude Couffon pero también la entrevista a Ernesto Sábato, su participación al número aniversario de Rubén Darío y, last but no least, la dirección del homenaje a Lezama Lima, se tendrá una idea del espacio que Mundo Nuevo fue para Sarduy, no solo de promoción de su literatura ni de expresión de teorías personales sino un lugar de ejercicio de la práctica crítica y de relectura de la tradición latinoamericana. 
de soledad (uno en 1966 y otro 1967), El gran sertón, El obsceno pájaro de la noche, Celestino antes del alba (Le puits) y dos de La traición de Rita Hayworth, la primera novela de Manuel Puig que antes de ser publicada por Gallimard en 1969 había sido evaluada y en cierta forma acompañada en su génesis por los asesores de Seuil (Camenen 2021).

La coincidencia de las publicaciones no es, desde luego, ningún azar y es en cierta forma la faz visible de la manera en que los textos circulaban por las redes informales, de amigos y passeurs y pasaban por diferentes estados de publicación antes de existir como libros. La repetición de nombres y títulos, además de un tránsito material de manuscritos, devela una visión crítica común, una lectura histórica de la novelística reciente. No es exagerado pensar en efecto que el catálogo de Cadre vert deba algo de su vertebración al célebre cuadro de la novelística latinoamericana que Monegal trazó en un congreso de literatura en Caracas en julio de 1967 y reprodujo en la revista poco después (Rodríguez Monegal 1967b). Recordemos que en este intento de historia reciente, que se difundió rápidamente hasta volverse canónico, Monegal reagrupaba en cuatro "promociones", sin seguir exactamente el método generacional de Ortega y Gasset, a una serie de autores desde los años cuarenta hasta el presente. La primera promoción, la de los "renovadores" reunía a Borges, Asturias, Carpentier, Yánez y Marechal quienes efectuaron la liquidación del naturalismo y la afirmación de la obra literaria como obra de ficción. Una vez esta conquista asegurada, la segunda promoción, la de Guimarães Rosa, Otero Silva, Onetti, Sábato, Lezama Lima, Cortázar y Rulfo desarrolló "una conciencia de la estructura de la novela y una sensibilidad para el lenguaje como materia prima de lo narrativo" ; la tercera incluía a Martínez Moreno, Lispector, Donoso, Fuentes, García Márquez, Cabrera Infante y Vargas Llosa que fueron “eficaces fabricantes de máquinas de novelar” y finalmente, la cuarta, la de los novísimos -Puig, Sánchez y Sarduy- se definía por su atención exclusiva al lenguaje como materia de su narrativa.

Un rápido cotejo de la lista de Cadre vert con el cuadro histórico de Monegal muestra que la colección francesa publicó los autores clasificados y por lo tanto destacados por Monegal, y entre ellos, a escritores pertenecientes a las cuatro promociones, proponiendo de este modo a su lector francés, y a su escala, una pequeña muestra de la historia reciente de la novela del continente escrita por la revista. Borges, editado en la selección y canonizado por la publicación del número de "Écrivains de toujours", en ambos casos con Monegal como autor, ocupaba en esta historia o sistema en miniatura el lugar de "criterio fundador de toda referencia a la novedad o calidad de un texto" que le confería la revista (Rodríguez-Carranza 1992: 910). Es decir que la colección encontró en la revista una lectura de la tradición latinoamericana moderna. 
La comparación devela sin embargo diferencias. Una de ellas es la importancia dada a la actualidad; criterio absoluto para Monegal, no lo era tanto como lo podían afirmar los editores de Seuil. Los escritores más representados en Mundo Nuevo pertenecían al tercer y al cuarto grupo del cuadro de Monegal pero en Cadre vert las cuatro promociones aparecían de manera relativamente equilibrada (Rodríguez-Carranza 1992: 909). Esta distribución más homogénea en la colección matiza el afán vanguardista de los editores, el que se expresaba en la encuesta de Bareiro Saguier y animaba el artículo de Sarduy sobre Cien años de soledad. La presencia en el catálogo de autores de diferentes generaciones, con estéticas más o menos convencionales o vanguardistas, muestra que las normas que regían la constitución del catálogo eran las de una colección de literatura extranjera que difícilmente podía especializarse en una línea exclusivamente vanguardista. La inclusión de Ernesto Sábato en la colección es el mejor ejemplo de este equilibrio entre generaciones y estéticas y, además, de las diferencias entre la revista y la colección con respecto a la evaluación de los libros. En efecto, a pesar del espacio que Monegal concedió a Sábato en Mundo Nuevo, mandó al editor norteamericano que había solicitado su opinión a propósito de Sobre héroes y tumbas un informe demoledor que fue un considerable freno para la publicación de la novela en los Estados Unidos, que solo se dio en 1981(Mudrovic 2012: 130). Otra divergencia concierne la valoración de lo nacional. Rechazado con una irritación casi militante como un lastre del pasado por un Monegal que fustigaba "el color local” y la "cultura de provincia” (Monegal 1966a. 7), lo nacional era, como lo sugerimos antes, un criterio de lectura válido y un argumento de seducción del lector de Cadre vert.

La imagen del filtro quizás sea la más adecuada para figurar la relación que unía Mundo Nuevo y Cadre vert. Ni operación mecánica de trasvase, ni pura emanación, pese a la coquetería de Sarduy, la colección separaba, recortaba y recomponía series de escritores y argumentos de presentación en función de su lector (el francés) de su soporte (el libro) y de su campo (la literatura extranjera en Francia). Valga como muestra otro cotejo, entre el texto de presentación de Celestino antes del alba en Mundo nuevo y el de la contraportada de Le puits, su traducción al francés. El primer texto es muy probablemente del mismo Monegal, ya que no solo la narrativa era coto del director de la revista sino que, en repetidas ocasiones, valoró la obra de Arenas; el segundo es la reproducción casi literal del informe de lectura de Sarduy para Seuil:

Una de las novelas más originales que se ha publicado en Cuba estos últimos años es, sin duda, Celestino antes del alba, de Reinaldo Arenas, cuya versión francesa publicarán próximamente las Editions du Seuil. Centrada en la visión de un niño idiota, cuyo monólogo constituye la materia prima del relato, la novela va revelando un mundo campesino a la vez brutal y tierno, grotesco y patético, onírico y realista. El aparente regionalismo del 
enfoque resulta superado no solo en la lengua y la anécdota, sino sobre todo en el curioso montaje de textos que ha efectuado Arenas para dar simultáneamente todos los niveles de una "realidad" alucinada que no reconoce las fronteras del tiempo o del espacio, que trasciende lo natural como lo sobrenatural, y que se apoya en definitiva sobre una única textura concreta y continua: la del lenguaje. Libro lleno de humor y fantasía, pero también circundado por el terror, Celestino antes del alba se inscribe en una tradición latinoamericana que tiene a José Lezama Lima de maestro y asimismo entronca con otros mágicos superadores del folklorismo, como Guimarães Rosa y García Márquez. (N. de la R.) (Arenas 1968: 34).

¿Quién es Celestino? Un poeta: escribe en cualquier superficie blanca que encuentre hasta en el tronco de los árboles. Un loco: no sabe hacer nada excepto soñar, llorar. Un niño campesino cubano que posa sobre todas las cosas una mirada que las vuelve fabulosas, como si en ello se le fuera la vida, en un universo hostil a los poetas, a los locos, a los niños.

Reinaldo Arenas, celebrado aquí con la publicación de El mundo alucinante como el mejor de los jóvenes novelistas de La Habana, emprende con este relato una obra en varios volúmenes, historia de una vida desde sus primeros pasos en un mundo casi inmemorial hasta la Revolución y la prefiguración de una época (contratapa de Le puits, Seuil, 1972).

El proceso de decantación que se observa de un texto al otro afianza una sustancia común, la apreciación de la novela de Arenas como transfiguración de la realidad por la fuerza de la imaginación, dejando aflorar, no obstante, sobre este núcleo compartido, las líneas de fuerza que distinguen una lectura de la otra: la superación del regionalismo y el lenguaje como única materia para la primera, el desesperado enfrentamiento de un individuo con un mundo hostil para la segunda.

No cabe duda de que el primero en advertir y comprender este proceso de decantación que modula el discurso crítico para encaminarlo por nuevos canales, fuera el propio Monegal. En su estudio sobre Octavio Paz, el director de Mundo Nuevo distinguía dos concepciones diferentes y complementarias de la crítica. Una consiste en la creación de un "doble" de la obra misma, en la atención a su infinita apertura y al trayecto de sus múltiples interpretaciones; la otra en la creación de un ámbito intelectual que establece vínculos entre los textos. No es casual, declaraba Monegal, que:

[. . .] algunos de los más importantes críticos de América Latina, desde Bello hasta Borges, pasando por Rodó y por Reyes, hayan sido no solo críticos sino promotores de cultura, hayan estado asociados a editoriales, hayan publicado revistas. No solo han creado su obra de críticos, poetas o narradores. Los cuatro han debido fundar una literatura (Rodríguez Monegal 1968: 56). 
Con su implicación en la promoción de los libros, su presencia activa en la prensa francesa, Sarduy, como Rubén Darío con Mundial magazine, abría el "espacio intelectual” latinoamericano, como lo hizo, por su lado, Monegal con su revista. Esta afinidad invita a pensar que Mundo Nuevo no fue la antesala que prepara a la entronización editorial ni el cenáculo cerrado de una “clericatura” (Fell 1990: 171). Fue, para Cadre vert, un café parisino donde se conversaba, con la pasión y el entusiasmo que destilan las entrevistas publicadas en Mundo Nuevo.

Es probable que el catálogo de lo que propusimos llamar "el momento latinoamericano" de Cadre vert haría palidecer de envidia a muchos editores de hoy. Las novelas que incluye, aunque con suertes desiguales, no solo gozan de la permanencia que confiere un destino académico, hecho de lecturas en aulas y congresos, sino que varias tienen el raro privilegio de prolongar su vida literaria en la obra de los escritores que reconocen en ellos a un predecesor, un pionero o incluso un maestro.

Para no dejarnos cegar por la luz del catálogo, quisimos, en el ejercicio de microhistoria que ahora concluimos, delinear los contornos de un objeto, la colección, que, postulamos, merece considerarse como un todo autónomo, aunque vinculado con las novelas que lo configuran, como las relaciones que unen las partes con el todo. Este objeto, al contrario de lo que imaginamos con ingenua esperanza al iniciar nuestra indagación, no salió de la cabeza genial de un editor visionario, como un programa estético y cultural definido, sino que fue el fruto de las circunstancias históricas que intentamos recomponer y de las contingencias de un negocio cuyas reglas iban cambiando con la afirmación de los agentes y, en especial, de su representante egregia y temida, Carmen Balcells. En otras palabras, el genio de los editores fue, como su obra, mixto: crítico, estratégico y, pese a ello, frenado en su ambición.

A modo de provisoria conclusión, diremos que el brillo de la selección de Durand y Sarduy en Cadre vert es el de una época cuyos rasgos, la efervescencia de la imaginación creadora y el impulso de la libertad crítica, encarnó como pocas. Pero compartió también con esta época otra característica: la politización extrema del campo intelectual y literario, cuyo estudio es necesario a su plena comprensión ${ }^{10}$. Con solo recordar que Cadre vert publicó en 1969 Hors jeu, el poemario que inició el primer “caso Padilla” acelerando la disgregación

10 Quedan por explorar numerosas zonas de esta selección como, por ejemplo, las traducciones de Durand, los vínculos de la reflexión estética de Sarduy con su actividad de editor o las cuestiones planteadas por la edición de cada autor, o incluso cada libro, de la selección. 
de los "lazos de familia” que, según Claudia Gilman, unían a los escritores desde hacía media década, se tiene una idea de que Seuil estaba en el ojo del huracán.

\section{Bibliografía}

Arenas, Reinaldo (1968): “Celestino antes del alba”. En: Mundo Nuevo 21, pp. 34-41.

Basuyaux, Marie-Laure (2004): “Les années 1950: Jean Cayrol et la figure de Lazare”. En: Fabula/Les colloques, L’idée de littérature dans les années 1950, <http://www.fabula. org/colloques/document61.php> [última visita: 12/01/2021].

Bareiro Saguier, Rubén (1968): “La literatura latinoamericana en Francia”. En: Mundo Nuevo 30, pp. 52-66.

Beuve-Mery, Alain (2016): “Carmen Perea Jiménez, traductrice, est morte à 78 ans”. En: Le monde, 3/02/2016.

Camenen, Gersende (2021): “De pop a naîf: la mediación editorial francesa de La traición de Rita Hayworth (1964-1969)”. En: Guerrero, Gustavo/ Loy, Benjamin/ Müller, Gesine: World Editors. Dynamics of Global Publishing and the Latin American Case between the Archive and the Digital Age. Berlin/Boston: De Gruyter, pp. 279-296.

Catelli, Nora (2010): "La élite itinerante del boom: seducciones transnacionales en los escritores latinoamericanos (1960-1973)”. En: Carlos Altamiraono (ed.): Historia de los intelectuales en América Latina II. Los avatares de la "ciudad letrada" en el siglo XX. Buenos Aires: Katz Editores, pp. 712-732.

Chaubet, François (2018): Claude Durand. Paris: Editions du Cerf.

De Diego, José Luis (2019): Los autores no escriben libros. Nuevos aportes a la historia de la edición. Buenos Aires: Ampersand.

Díaz Martínez, Liset (2021): “Mapping Gabo editors, introducción al estudio de la correspondencia en los archivos editoriales". En: Guerrero, Gustavo/Camenen, Gersende (eds.): La literatura latinoamericana en versión francesa. Berlin/Boston: De Gruyter, pp. 273-295.

Dosse, François (2014): Les hommes de l'ombre. Portraits d'éditeurs. Paris: Perrin.

Durand, Claude (2011): Agent de Soljénitsyne. Paris: Fayard.

Fell, Claude (1990): “La revue Mundo Nuevo, catalyseur du “boom” latino-américain”. En: Cahiers de l'UFR d'Etudes Ibériques et Latino-américaines, pp. 163-172.

- (1992) "La Croix du Sud: tremplin de la littérature latinoaméricaine en France". En: Rio de la Plata 13-14, pp. 173-189.

Forest, Philippe (1995): Histoire de Tel Quel 1960-1982. Paris: Seuil.

Gilman, Claudia (2003): Entre la pluma y el fusil. Debates y dilemas del escritor revolucionario en América Latina. Buenos Aires: Siglo XXI editores.

Gras, Dunia (2000): “Barcelona, plataforma cultural de América Latina en Europa”. En: Riquer, Isabel/ Losada, Elena/González, Helena (eds.) Basilio Losada: Ensinar a pensar con liberdade e risco. Barcelona: Publicacions de la Universitat de Barcelona, pp. 445-451.

- (2020): “Transatlantic Literary Networks during the Cold War: Emir Rodríguez Monegal, reader for Gallimard" En: Territories: A Trans-Cultural Journal of Regional Studies 2. <http://dx.doi.org/10.5070/T22144885>. 
Guerrero, Gustavo (2018): “La Croix du Sud (1945-1970): génesis y contextos de la primera colección francesa de literatura latinoamericana”. En: Müller, Gesine/ Locane, Jorge J. /Loy, Benjamin (eds.): Re-mapping World Literature: Writing, Book Markets and Epistemologies. Berlin/Boston: De Gruyter, pp. 199-208.

- (2021): “José Lezama Lima en Francia: edición, traducción, difusión”. En: Guerrero, Gustavo/Camenen, Gersende (eds.): La literatura latinoamericana en versión francesa. Berlin/Boston: De Gruyter, pp. 219-245.

Hage, Julien (2011): “L'édition européenne sur les chemins de l'Amérique latine en lutte”. En: Mollier, Jean-Yves/Vallotton, François/ Vincent, Josée (eds.), La diplomatie par le livre. Paris: Nouveau Monde éditions, pp. 363-378.

Lindon, Mathieu (1999): “Claude Durand, l'homme-orchestre. PDG de Fayard, il a traduit et publié Cent ans de solitude de García Márquez et est devenu l'agent de Soljenitsyne”. En: Libération, 28 de julio de 1999, p. 7.

Louis, Annick (2013): “Etoiles d'un ciel étranger. Roger Caillois et l'Amérique latine”. En: Littérature 170, pp. 71-81.

McQuade, Franck (1992): “Mundo Nuevo: la nueva novela y la guerra fría cultural”. En: América: Cahiers du Criccal 9-10, pp. 17-26.

Morejón, Idalia (2017): Política y polémica en América Latina. Las revistas 'Casa de las Américas' y 'Mundo Nuevo'. Leiden: Almenara.

Mudrovic, María Eugenia (1997): Mundo Nuevo. Cultura y Guerra Fría en la década del 60. Rosario: Beatriz Viterbo.

- (2002): "Reading latin american literature abroad: agency and canon formation in the sixties and seventies". En: Balderston, Daniel/Schwartz Marcy E. (eds): Voice-Overs: Translation and Latin American Literature, Albany: State University of New York pp. 129-143.

Nadeau, Maurice (1970): Le roman français depuis la guerre. Paris: Gallimard.

Parinet, Elisabeth (2004): Une histoire de l'édition à l'époque contemporaine XIX-XXe siècle. Paris: Seuil.

Rama, Ángel (1984): El boom en perspectiva. En: Viñas, David (ed): Literatura y mercado, Buenos Aires: Folio Ediciones, pp. 51-110.

Rodríguez Monegal, Emir (1966a): "Situación del escritor en América latina (diálogo con Carlos Fuentes)”. En: Mundo Nuevo 1, pp. 5-21.

- (1966b): "Las estructuras de la narración (diálogo con Severo Sarduy)”. En: Mundo Nuevo 2, pp. 14-26.

- (1966c): “Por una novela novelesca (diálogo con Ernesto Sábato)”. En: Mundo Nuevo 5, pp. 5-21.

- (1967b): “Diario de Caras”. En: Mundo Nuevo 17, pp. 4-24.

- (1968): “Octavio Paz. Crítica y poesía”. En: Mundo Nuevo 20, pp. 55-63.

- (1969): “Préface”. En: Borges, Jorge Luis: Evaristo Carriego, traducido del español por Marie-Françoise Rosset. Paris: Seuil, pp. 5-8.

- (1970): Borgès par lui-même, traducción de Marie-Françoise Rosset. Paris: Seuil, colección “Écrivains de toujours", $n^{\circ} 86$.

Rodríguez-Carranza, Luz (1992): “Emir Rodríguez Monegal o la construcción de un mundo (nuevo) posible”. En: Revista iberoamericana 160-161, pp. 903-917.

Sapiro, Gisèle (2019): “Les grandes tendances du marché de la traduction”. En: Banoun, Bernard/ Poulin, Isabelle/Chevrel, Yves (eds.): Histoire des traductions en langue française XXe siècle. Paris: Verdier, pp. 55-176. 
Sarduy, Severo (1968): “L'écriture autonome”. En: La Quinzaine littéraire 63, pp. 3-4.

Serry, Hervé (2002): “Constituer un catalogue littéraire”. En: Actes de la recherche en sciences sociales 144, pp. $70-79$.

- (2005): "Figures d'éditeurs français après 1945: habitus, habitus professionnel et transformation du champ editorial”. En: Legendre, Bertrand/Robin, Christian (eds.):

Figures de l'éditeur. Représentations, Savoirs, Compétences, Territoires. Paris: Nouveau Monde Edition, pp. 73-89.

- (2006): “L'essor des Editions du Seuil et le risque littéraire. La création de la collection 'Fiction et Cie'”. En: Bessard-Banquy, Olivier (ed.): L'édition littéraire aujourd'hui. Rennes: PUB, pp. 163-190.

Simonin, Anne (1998): “L'édition littéraire”. En: Fouché, Pascal (ed.): L'édition française depuis 1945. Paris: Editions du cercle de la librairie, pp. 31-88.

Spiers, John (2011): "Introduction: wondering about the "causes of causes": the publisher's series, its cultural work and meanings". En: Spiers, John (eds): The Culture of the Publisher's series. Vol. 1. Authors, publishers and the shaping of taste. London: Palmgrave Macmillan.

Steenmeijer, Marteen (2002): "How the West Was Won: Translations of Spanish American Fiction in Europe and the United States". En: Balderston, Daniel/Schwartz Marcy E. (eds): Voice-Overs: Translation and Latin American Literature, Albany: State University of New York, pp. 144-155.

Tuñon de Lara, Manuel (1958): “Initiation à l'Amérique latine”. En: Esprit 266, pp. 307-310. Winock, Michel (1987): Chronique des années soixante. Paris: Seuil, coll. Points/Histoire. - (2011): L'effet de génération, une brève histoire des intellectuels français. Vincennes: Editions Thierry Marchaisse. 